\title{
Towards instantaneous quantitative fluoroimaging drugs determination in body fluids with no added reagents
}

\author{
Natalia V. Strashnikova ${ }^{\text {a }}$, Arcady P. Gershanik ${ }^{\text {a }}$, Nona Papiashvili ${ }^{\text {a }}$, Daniel Khankin ${ }^{\text {, }}$, \\ Rivka Cohen-Luria ${ }^{\mathrm{a}}$, Shlomo Mark ${ }^{\mathrm{b}}$, Yehoshua Kalisky ${ }^{\mathrm{c}}$ and Abraham H. Parola ${ }^{\mathrm{a}, *}$ \\ ${ }^{a}$ Department of Chemistry, Ben Gurion University, Beer Sheva, Israel \\ ${ }^{\mathrm{b}}$ Department of Software Engineering, Shamoon College of Engineering, Beer Sheva, Israel \\ ${ }^{\mathrm{c}}$ Chemistry Department, Nuclear Research Center Negev, Beer Sheva, Israel
}

\begin{abstract}
Our objective is to develop a simple monitoring technique for rapid, sensitive and quantitative drugs detection in body fluids, with no reagent added and no need for qualified professionals. The user-friendly automatic Fluo-imager will (a) measure the full-range 3D fluorescence map of the inserted fluid sample, (b) determine the chemical nature and concentration of the drugs and (c) transfer the results through internet to the diagnosis center. For these goals the fluorescence measurement data will be examined by neuronal network-pattern recognition software. The software identifies the chemical nature and the appropriate concentration of the drug by comparison of the obtained 3D pattern with the contents of the data bank. One of the problems in the approach under consideration is the high optical density of body fluids in the UV region, which raises difficulties in the fluorescence measurements. In this paper, we have attempted to overcome this problem by means of preliminary dilution. Nevertheless, the problem of subtraction of the fluid fluorescence background still needs to be addressed.
\end{abstract}

Keywords: Therapy, drug, monitoring, Fluo-imager, 3D fluorescence, fluorescence spectra, pattern recognition software, data bank, diluted human plasma, concentration-calibration curves

\section{Introduction}

Therapeutic drug monitoring (TDM) is an important and permanently growing field of clinical medicine. In addition, monitoring of the antibiotics in blood is one of the well-known tasks of TDM due to the continuous growth of bacterial resistance $[1,5,6]$. The problems presently associated with the clinical TDM are the following: (i) testing requires regional or hospital laboratories, (ii) relatively lengthy delays in obtaining results, (iii) long distance transfer of blood, urine, etc., samples (including air shipments), (iv) the need for laboratory technicians and special reagents, (v) testing requires professional expertise and (vi) expensive procedures and heavy-duty equipments. The most common analytical methods in TDM are immunoassays and HPLC techniques which entail specific procedures for each drug analysis. Therefore, these procedures are complex, difficult to automate and are hard to unify.

Our objective is to develop a user-friendly fully automated procedure capable of monitoring qualitatively, quantitatively and simultaneously the presence of multitude drugs and toxic substances in body fluids. The method should be sensitive, highly-accurate, rapid, reliable and inexpensive.

\footnotetext{
*Corresponding author: Prof. Abraham H. Parola, Department of Chemistry, Ben Gurion University, P.O. Box 653, Beer Sheva, 84105, Israel. Fax: +972 8647 2943; E-mail: aparola@bgu.ac.il.
} 
We have chosen fluorescence spectroscopy because of its uniquely high sensitivity. The well-known problem of substance identification by fluorescence spectroscopy may gain considerably by utilizing 3D fluorescence imaging, in which the fluorescence intensity is plotted simultaneously against the excitation and emission wavelengths in all the range of available wavelengths. This would allow both the qualitative and quantitative determination of the components in the analyzed mixture.

The presently available automatic computerized Fluo-imager performs the scanning in a wide ranges of excitation (240-360 nm) and emission (265-580 nm) wavelengths within about 1 min only. Our efforts were aimed at testing the Fluo-imager as an adequate solution for this automatic multipurpose analysis.

\section{Experimental}

\subsection{The Fluo-imager}

PC controlled 3D-spectrofluorimeters (Fluo-imagers), models M50 and M50C for liquid samples, have been designed and built according to our specification by SKALAR-LDI Ltd. A continuous $150 \mathrm{~W}$ and a pulsed $15 \mathrm{~W}$ quartz Xe-lamp serve as the light source in models M50 and M50C, respectively. The Fluo-imager M50C is equipped with a monochromator (driven by a step motor), holographic diffraction grating, and with an intensified linear 500 pixels CCD-detector. A microcontroller provides the control of measurement cycle, storage of data, preliminary data processing and data transfer to the $\mathrm{PC}$ via standard Serial Port.

The mode of measurement is a classical transmission mode and the compact geometry renders a short light path which results in the high sensitivity. Data treatment utilizes a special substance identification algorithm which renders a quick response and short data accumulation period.

\subsubsection{D fluorescence spectra}

The 3D fluorescence spectra are obtained automatically as follows. The initial excitation wavelength $(240 \mathrm{~nm})$ is transferred by the monochromator through a transparent $1 \mathrm{~cm}$ by $1 \mathrm{~cm}$ square quartz cuvette containing the liquid sample to be analyzed; the excitation beam is orthogonal to the front plane of the cuvette. The fluorescence response of the sample is taken at an angle of $90 \mathrm{deg}$. to the excitation beam and directed to the holographic diffraction grating and further to the CCD-array detector. Thus, each pixel receives from the grating a narrow band of the emission wavelength. Thus, due to its spatial position, each pixel detects a single narrow band. The combined output of the pixel array renders the complete emission spectrum at a given excitation wavelength. Next, the excitation wavelength is advanced by a single pre-dermined step. The CCD-array responds with a new emission spectrum, and the process continues for the whole range of excitation wavelengths. The combined output leads to the so called 3D spectra (e.g., Fig. 1). As indicated above, the overall 3D spectrum is attained within 1 min.

\subsubsection{Software}

The Fluoimager M50C program has been developed as a two iteration processes of three steps each: alpha, beta and release. The first iteration module serves as an independent confirmation tool. After a Software Test Plan, Description and Report [4] the program moved to C\# under Visual Studio .NET 2005, and every module passed all the testing phases (requirements, design, program, installation and regression testing) [3,7]. User feedback, suggestions and accuracy assessments were incorporated into the package, which was subjected to regression testing to make sure none of the improvements cause earlier passed tests to fail. 

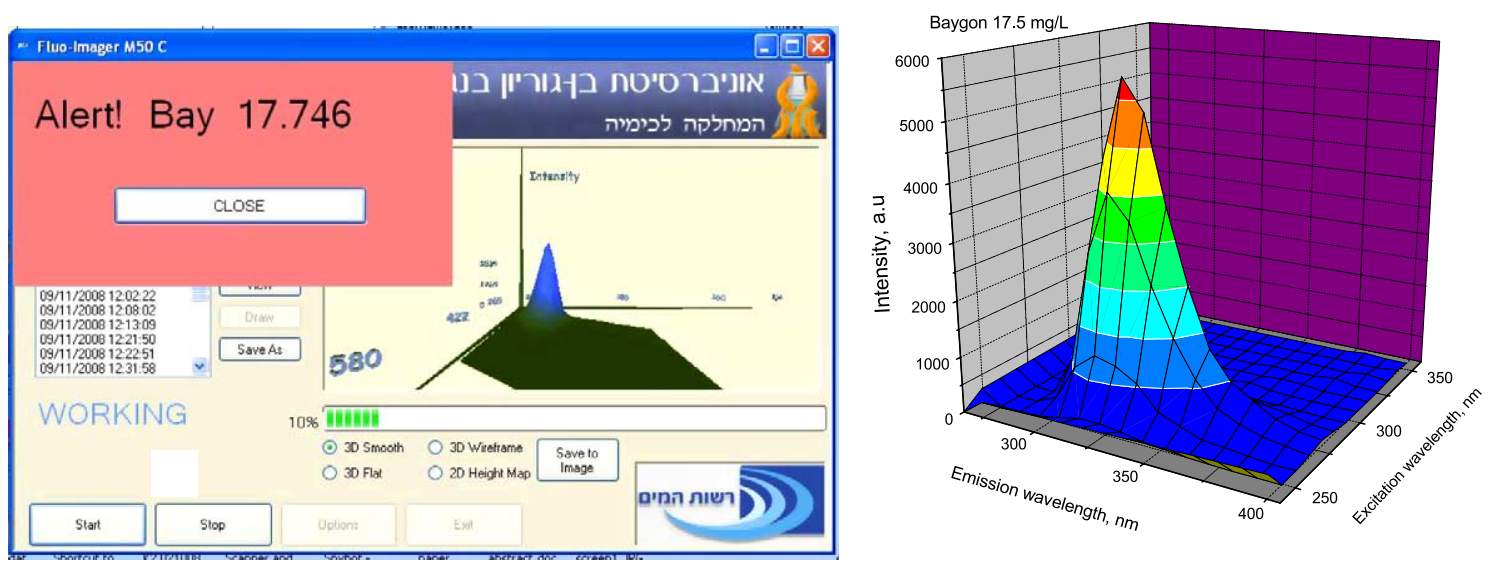

Fig. 1. A representative Fluo-imager M50 screen at the end of the measuring cycle warning about the detection of baygon (=Bay), $17.746 \mathrm{mg} / \mathrm{l}$, in water; the corresponding 3D spectrum is shown too. On the right, the 3D spectrum of this measurement which was simultaneously obtained for this solution prepared by dissolving $17.5 \mathrm{mg} / \mathrm{l}$ of baygon in water. The spectrum is saved in the memory of the remote control PC.

The analysis unit - SPECTRALYZER, was created specifically for analyzing the spectra. The idea beyond this independent unit is that it is not enough to use one method to find the various substances under various conditions [2]. This unit was designed to have the ability to readily use multiple methods for analyzing spectra, improving the chances of accurately detecting the important markers. The SPECTRALYZER was developed as neuronal network-pattern recognition software in order to identify and measure the concentration of the substances during analysis. The identification is made by comparing the obtained spectrum with the preliminary pre-gathered bank of spectra that contains the 3D images and the quantitative calibration data as well. This data bank is stored in the PC memory and can be easily exchanged with a remote central computer. Thus, the Fluo-imager may easily be integrated into a large system of analysis, e.g., a national service bank.

Spectra may be manipulated by this program in many different ways: subtracted, shifted, scaled and "smoothed", as well as peak matching. The user may determine which kind of preferred background spectrum should be subtracted for better quantitative results.

\subsection{Materials}

Drugs to be tested were obtained from the pharmacy at the Soroka Medical Center. Others were purchased from Sigma-Aldrich LTD, Riedel-de Haen or Merk. Insecticides were obtained from either Machteshim LTD or purchased from Aldrich.

Samples where either dissolved in doubly distilled water (DDW) or in 1:100 plasma or serum in DDW. Plasma was purchased from the blood bank of Soroka Hospital and serum was separated from blood samples donated by healthy donors. Typically, for a compound in powder, $5 \mathrm{mg}$ were dissolved in $5 \mathrm{ml}$ of the examined solvent. For bottled drugs or those in ampoules, the initial concentration was registered on them. Compounds with reduced solubility were vigorously stirred at elevated temperature, below $50^{\circ} \mathrm{C}$.

For fluorescence measurements, solutions at concentrations ranging from $2.0 \times 10^{-2}$ to $200.0 \mu \mathrm{g} / \mathrm{ml}$ or saturated solutions (their exact concentration was determined spectrophotometrically) have been measured at room temperature. For absorption studies, the HP, UV/VIS spectrophotometer, model 8452A 
and Jasco, model V-550 were used. The linear range of absorption obtained at variable concentrations was sought. Unless otherwise stated, the $\mathrm{pH}$ was kept at 6-7. Background signal of the solvent/diluted plasma was subtracted from the sample fluorescence or absorption spectrum.

\subsection{Instrument calibration}

The Fluoro-imager exhibits different sensitivity to various fluorophores, depending on their fluorescence quantum yield. Therefore we have pre-calibrated the Fluoro-imager with each of the studied fluorophores at several concentrations. The 3D spectra obtained during this calibration procedure were inserted into a specific catalogue which is than treated by the software. This software defines the calibration constants from the absolute maxima of the 3D spectra. Based on that, in the actual measurement of an unknown, it reveals on the screen its chemical identity and concentration, as shown in Fig. 1. The results of such a quantitative determination of the unknowns are given in Table 1 and the excellent correlation factors are shown in Fig. 2.

\subsection{Measurements}

Prior to the more complicated measurements in body liquids, we have checked the reliability and accuracy of the Fluo-imager by analyzing several fluorophores in aqueous solutions. We prepared solutions of several known concentrations of each fluorophore, measured their 3D spectra which were inserted into the Fluo-imager data bank. We then prepared similar "unknown" solutions to be qualitatively identified and quantitatively determined.

Following the proof of concept in aqueous solutions, we initiated drug analysis in plasma.

Different from aqueous solutions, human plasma has several complicating properties for fluorescence analysis. Probable drug modification by body fluids, variability in $\mathrm{pH}$ affecting the emission spectra and relatively high absorption in the UV region are among these complicating properties. We intend to overcome these problems. For instance, drug modification by body fluids, when occurs, would require the enlargement of the data bank and would be considered as new substances. In cases where a $\mathrm{pH}$ dependence is anticipated (based on preliminary screening), one can include a $\mathrm{pH}$-meter signal with the automatic measurement cycle as well as with the software, which will be applied only to $\mathrm{pH}$ dependent drugs. Presently, in order to overcome the problem of high optical density of the plasma we study 1:100 diluted plasma. Its fluorescence background is subtracted from the 3D spectra of the added drug.

The problem of the background rising from diluted body liquids of healthy, untreated individuals would be solved in the future after careful learning of the spectral characteristics of the diluted plasma. One possibility is decoding of the nature of each background peak and resolving it by adequate software. An additional approach is the evaluation of the use of front surface spectroscopy specifically for opaque body fluids.

\section{Results}

The results of the Fluo-imager testing of fluorophores in aqueous solution are given in Table 2. The high reliability of substance identification is evident. Figure 2 depicts two examples of the quantitative determination of these substances.

The results of the analysis of drugs in plasma are given in Table 1. A 3 component mixture was correctly analyzed both qualitatively and quantitatively. Note that the concentrations in the table are given as measured, i.e., in diluted plasma. 
Table 1

The reliability of unknown substance identification and its concentration determination by the Fluoro-imager software

\begin{tabular}{|c|c|c|c|c|}
\hline$N$ & Solution in water ${ }^{1}$ & $\begin{array}{c}\text { Prepared/average } \\
\text { measured } \\
\text { concentrations }^{2}\end{array}$ & $\begin{array}{l}\text { Reliability factor } \\
\text { for qualitative } \\
\text { determination }^{3}\end{array}$ & $\begin{array}{l}\text { Correlation coefficient } \\
\text { for quantitative } \\
\text { determination }^{4} \\
\end{array}$ \\
\hline 1 & Aminocarb & $\frac{20.0}{20.4} \frac{2.5}{2.2} \frac{0.6}{0.8}$ & $1.00 ; 1.00 ; 0.95$ & 0.995 \\
\hline 2 & Aniline & $\frac{22.0}{22.6} \frac{2.7}{2.6} \frac{0.3}{0.2}$ & $1.00 ; 1.00 ; 0.90$ & 0.995 \\
\hline 3 & Asulam & $\frac{24.0}{23.5} \frac{3.0}{3.1} \frac{0.2}{0.4}$ & $1.00 ; 0.95 ; 0.95$ & 0.917 \\
\hline 4 & Bendiocarb & $\frac{26.0}{25.7} \frac{6.5}{7.0} \frac{3.0}{2.8}$ & $1.00 ; 1.00 ; 0.90$ & 0.999 \\
\hline 5 & Desmedipham & $\frac{3.0}{3.3} \frac{0.7}{1.0}$ & $1.00 ; 0.95$ & 0.995 \\
\hline 6 & 3,5-Dimethylphenol & $\frac{20.0}{18.8} \frac{1.2}{1.0} \frac{0.1}{0.05}$ & $1.00 ; 1.00 ; 0.95$ & 0.999 \\
\hline 7 & Ethoxyquin & $\frac{3.0}{2.7} \frac{0.4}{0.3} \frac{0.05}{0.02}$ & $1.00 ; 0.95 ; 0.95$ & 0.999 \\
\hline 8 & Baygon & $\frac{30.0}{28.6} \frac{2.0}{1.5} \frac{0.1}{0.06}$ & $1.00 ; 0.95 ; 0.90$ & 0.999 \\
\hline 9 & Carbofuran & $\frac{50.0}{50.5} \frac{6.2}{6.0} \frac{1.5}{1.9}$ & $1.00 ; 0.95 ; 0.90$ & 0.999 \\
\hline 10 & Methyl cotnion (Azinphos-methyl) & $\frac{10.0}{10.4} \frac{5.0}{4.8} \frac{2.5}{2.1}$ & $1.00 ; 1.00 ; 1.00$ & 0.946 \\
\hline 11 & Naphthalene & $\frac{15.0}{15.3} \frac{3.7}{4.0} \frac{0.1}{0.2}$ & $1.00 ; 0.95 ; 0.90$ & 0.985 \\
\hline 12 & Phenol & $\frac{6.0}{5.9} \frac{1.0}{1.0} \frac{0.06}{0.03}$ & $1.00 ; 1.00 ; 0.95$ & 0.999 \\
\hline 13 & Acenaphthenequinone & $\frac{10.0}{11.0} \frac{5.0}{5.4} \frac{1.0}{0.7}$ & $1.00 ; 1.00 ; 1.00$ & 0.995 \\
\hline 14 & $\begin{array}{l}\text { 1,2-Naphthoquinone-4-sulfonic acid } \\
\text { sodium salt }\end{array}$ & $\frac{20.0}{19.2} \frac{2.5}{2.1} \frac{0.3}{0.2}$ & $1.00 ; 0.95 ; 0.90$ & 0.995 \\
\hline 15 & Indole 3 Acetic Acid & $\frac{2.0}{1.9} \frac{0.5}{0.6} \frac{0.06}{0.07}$ & $1.00 ; 1.00 ; 0.90$ & 0.995 \\
\hline 16 & Estriol & $\frac{100.0}{88.2} \frac{12.5}{12.0} \frac{1.5}{1.3}$ & $1.00 ; 1.00 ; 0.95$ & 0.999 \\
\hline 17 & 17 Estradiol & $\frac{100.0}{93.6} \frac{25.0}{24.1} \frac{6.2}{5.5}$ & $0.95 ; 0.95 ; 0.90$ & 0.985 \\
\hline 18 & Fluorene & $\frac{1.0}{1.1} \frac{0.5}{0.4} \frac{0.1}{0.07}$ & $1.00 ; 1.00 ; 1.00$ & 0.995 \\
\hline 19 & 2-Cyanophenol & $\frac{10.0}{9.4} \frac{5.0}{4.5} \frac{1.2}{1.0}$ & $0.95 ; 1.00 ; 0.90$ & 0.957 \\
\hline 20 & Azoxystrobin & $\frac{20.0}{20.6} \frac{10.0}{10.3} \frac{2.5}{2.6}$ & $1.00 ; 1.00 ; 1.00$ & 0.999 \\
\hline 21 & Fenpropidin & $\frac{66.0}{65.0} \frac{13.5}{12.8}$ & $1.00 ; 0.90$ & 0.995 \\
\hline
\end{tabular}


Table 1

(Continued)

\begin{tabular}{|c|c|c|c|c|}
\hline$N$ & Solution in water ${ }^{1}$ & $\begin{array}{c}\text { Prepared/average } \\
\text { measured } \\
\text { concentrations }\end{array}$ & $\begin{array}{l}\text { Reliability factor } \\
\text { for qualitative } \\
\text { determination }^{3}\end{array}$ & $\begin{array}{c}\text { Correlation coefficient } \\
\text { for quantitative } \\
\text { determination }^{4} \\
\end{array}$ \\
\hline 22 & $\begin{array}{l}\text { 1-Naphthol-3,6-disulfonicacid, } \\
\text { disodium salt hydrate }\end{array}$ & $\frac{12.5}{12.7} \frac{3.1}{3.4} \frac{0.8}{1.3}$ & $1.00 ; 1.00 ; 0.95$ & 0.999 \\
\hline 23 & 4-Fluorophenol & $\frac{10.0}{10.1} \frac{2.5}{2.7} \frac{0.3}{0.3}$ & $1.00 ; 1.00 ; 0.95$ & 0.995 \\
\hline 24 & 4-Chlorophenol & $\frac{12.0}{11.5} \frac{3.0}{2.7} \frac{0.4}{0.3}$ & $1.00 ; 0.95 ; 0.95$ & 0.968 \\
\hline 25 & 1-Naphthol & $\frac{12.0}{12.6} \frac{1.5}{1.8} \frac{0.2}{0.3}$ & $1.00 ; 1.00 ; 1.00$ & 0.995 \\
\hline 26 & Toluene & $\frac{86.6}{90.0} \frac{11.0}{10.8}$ & $0.95 ; 0.95$ & 0.814 \\
\hline
\end{tabular}

Notes: ${ }^{1}$ Tapped water at neutral $\mathrm{pH}$ and room temperature $;{ }^{2}$ unknown solution prepared at the relevant soluble concentration $(\mu \mathrm{g} / \mathrm{ml})$, nominator, were prepared and their concentration was determined (in $\mu \mathrm{g} / \mathrm{ml}$ ) by the Fluo-imager, denominator, based on the pre-prepared data bank and the concentration calibration curves; ${ }^{3}$ defined as the ratio of correct identifications divided by the total number of repeat independent attempts (between 10 and 20). In most cases of the substance identification dismissing it was found operator's mistake; ${ }^{4}$ the correlation coefficients of the linear curves obtained by plotting the measured against the predetermined concentrations.

Indole 3 Acetic Acid

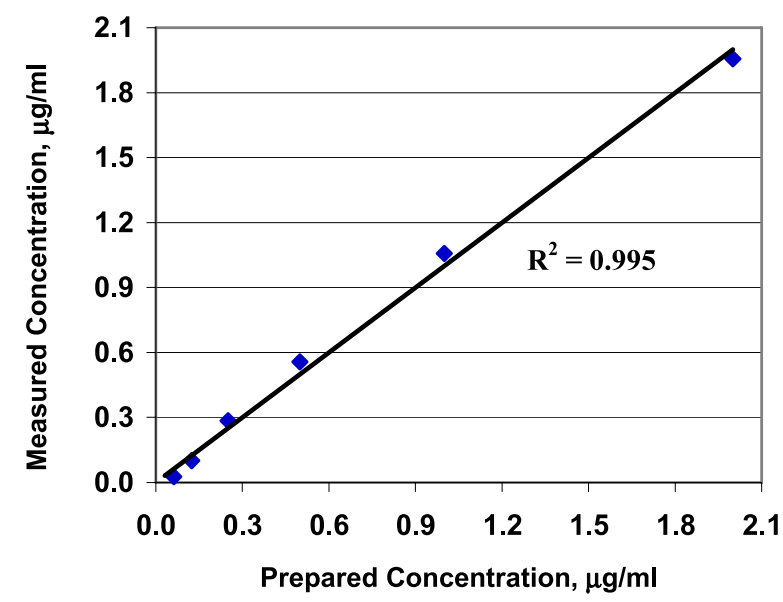

Phenole

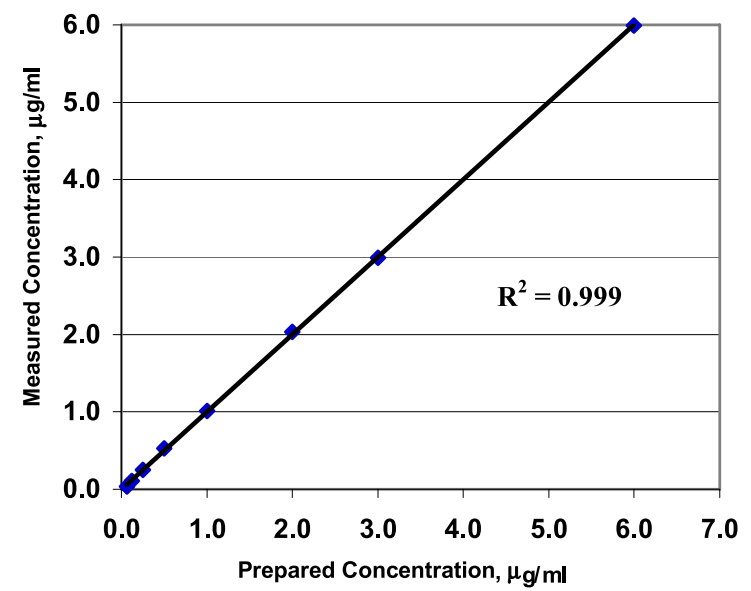

Fig. 2. Quantitative determination of indol-3-acetic acid and phenol in water.

\section{Discussion}

The inherent compactness, reliability and wide functional flexibility of the designed Fluo-imager prototype promise high perspectives for its suitability to TDM.

The instrument displays on the screen shows the chemical nature of the detected substance, its concentration and further shows the overall 3D view of the spectrum. 
Table 2

Quantitative determination of individual fluorophores in mixed samples: determining of drugs in diluted human plasma

\begin{tabular}{lcccccccc}
\hline $\begin{array}{l}\text { Solution of the } 3 \text { components } \\
\text { in diluted plasma }\end{array}$ & \multicolumn{7}{c}{ Prepared vs. average measured concentrations } \\
\hline Na-salicylate, $\mathrm{ng} / \mathrm{ml}$ & Prepared & 48.0 & 96.0 & 96.0 & 192.0 & 192.0 & 192.0 & 48.0 \\
& Measured & $48.4 \pm 0.8$ & $94.3 \pm 1.7$ & $96.3 \pm 0.3$ & $192.3 \pm 0.2$ & $192.3 \pm 0.2$ & $191.8 \pm 0.1$ & $48.8 \pm 1.8$ \\
Dobutamine, $\mu \mathrm{g} / \mathrm{ml}$ & Prepared & 5.0 & 10.0 & 20.0 & 50.0 & 50.0 & 5.0 & 50.0 \\
& Measured & $5.0 \pm 0.1$ & $10.0 \pm 0.1$ & $20.0 \pm 0.1$ & $50.0 \pm 0.1$ & $50.0 \pm 0.1$ & $5.0 \pm 0.1$ & $50.0 \pm 0.1$ \\
Quinidine, $\mathrm{ng} / \mathrm{ml}$ & Prepared & 78.0 & 78.0 & 156.0 & 312.0 & 78.0 & 312.0 & 312.0 \\
& Measured & $78.0 \pm 1.2$ & $76.7 \pm 1.8$ & $153.1 \pm 1.8$ & $312.0 \pm 0.3$ & $78.0 \pm 0.6$ & $312.7 \pm 0.2$ & $311.8 \pm 0.6$ \\
\hline
\end{tabular}

The pre-prepared mixtures of three drugs, sodium salicylate at the $\mathrm{ng} / \mathrm{ml}$ range, dobutamine at the $\mu \mathrm{g} / \mathrm{ml}$ range and quinidine at the $\mathrm{ng} / \mathrm{ml}$ range, all at physiologically relevant concentrations, were tested in human plasma (1:100 diluted by DDW) by our fluoroimaging method and revealed the expected concentration values. It is conceivable that further studies addressing the above mentioned difficulties should result in the successful implementation of this approach to TDM.

The advantages of the method make it unique: there is no need for pre-treatment of the sample and no use of antibodies. The method is specific and instantaneous, highly sensitive and enabling both quantitative and qualitative identification of fluorescent unknowns. It provides simultaneous identification of multitude components and enables real time data transfer.

The Apparatus is inexpensive, compact and user-friendly and does not require any special operation know-how. It can be set for continuous flow analysis as well as field work.

The following implementation is anticipated: the device will be freely distributed and reimbursement will be made upon delivery of the analysis results at much reduced rates than presently charged. It will be based on the continuous dynamic expansion of the data bank and results will be delivered through the internet rather than depend on air shipment of test samples.

The anticipated success of the proposed determination of drugs in body fluids will allow use not only in hospital laboratories, but will also include bed-side testing and MD's office diagnostics. Furthermore, since it is quick and simple it will allow the screening of large populations for drug abuse (e.g., high school students, army and industrial personnel, drivers, etc.).

The extension of the databank to include all fluorescent drugs and their pharmacokinetics and the development of mass production of cheap, miniaturized instrument (based on the existing prototype) are essential to the successful implementation of this approach. Moreover, the development of improved software for qualitative and quantitative identification is an essential on going effort.

\section{Acknowledgements}

We are grateful to Dr. Vitaly Sominsky, PhD, and Dr. Shlomo Almog, $\mathrm{PhD}$, for enabling the calibration tests of the narcotics at the Sheba Medical Center and to the Israeli Police for providing us with them. We thank for the fruitful discussions with Professor Yona Amitai, MD, and Razi Efron. 


\section{References}

[1] D.J. Birkett, Therapeutic drug monitoring, Austr. Prescr. 20(9) (1997).

[2] M. Fowler, Analysis Patterns, Addison-Wesley, 1997.

[3] A. Ireland, Software Engineering 4: The Software Testing Life-Cycle, School of Mathematical and Computer Sciences, Heriot-Watt University, Edinburgh, 2007.

[4] J. Radatz, M. Olson and S. Campbell, MIL-STD-498, Logicon, San Diego, CA, 1995.

[5] E. Schütz, M. Shipkova, V. Armstrong, P. Niedmann, L. Weber, B. Tönshoff, K. Pethig, T. Wahlers, F. Braun and B. Ringe, Therapeutic drug monitoring of mycophenolic acid: comparison of HPLC and immunoassay reveals new MPA metabolites, Transplantation Proceedings 30(4) (1998), 1185-1187.

[6] I. Sunshine, Recent Developments in Therapeutic Drug Monitoring and Clinical Toxicology, Marcel Dekker, New York, 1992.

[7] J. Watkins (ed.), Testing IT: An Off-the-Shelf Software Testing Process, Cambridge University Press, Cambridge, 2001. 


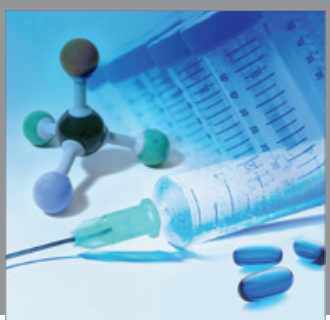

International Journal of

Medicinal Chemistry

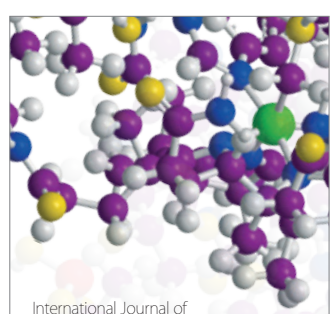

Carbohydrate Chemistry

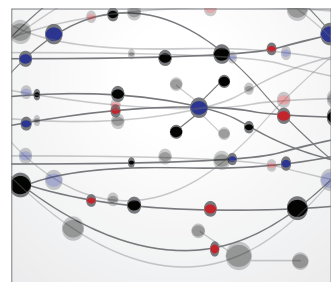

The Scientific World Journal
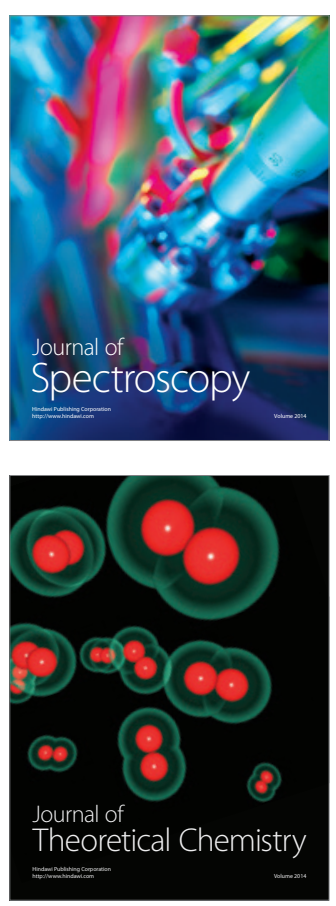
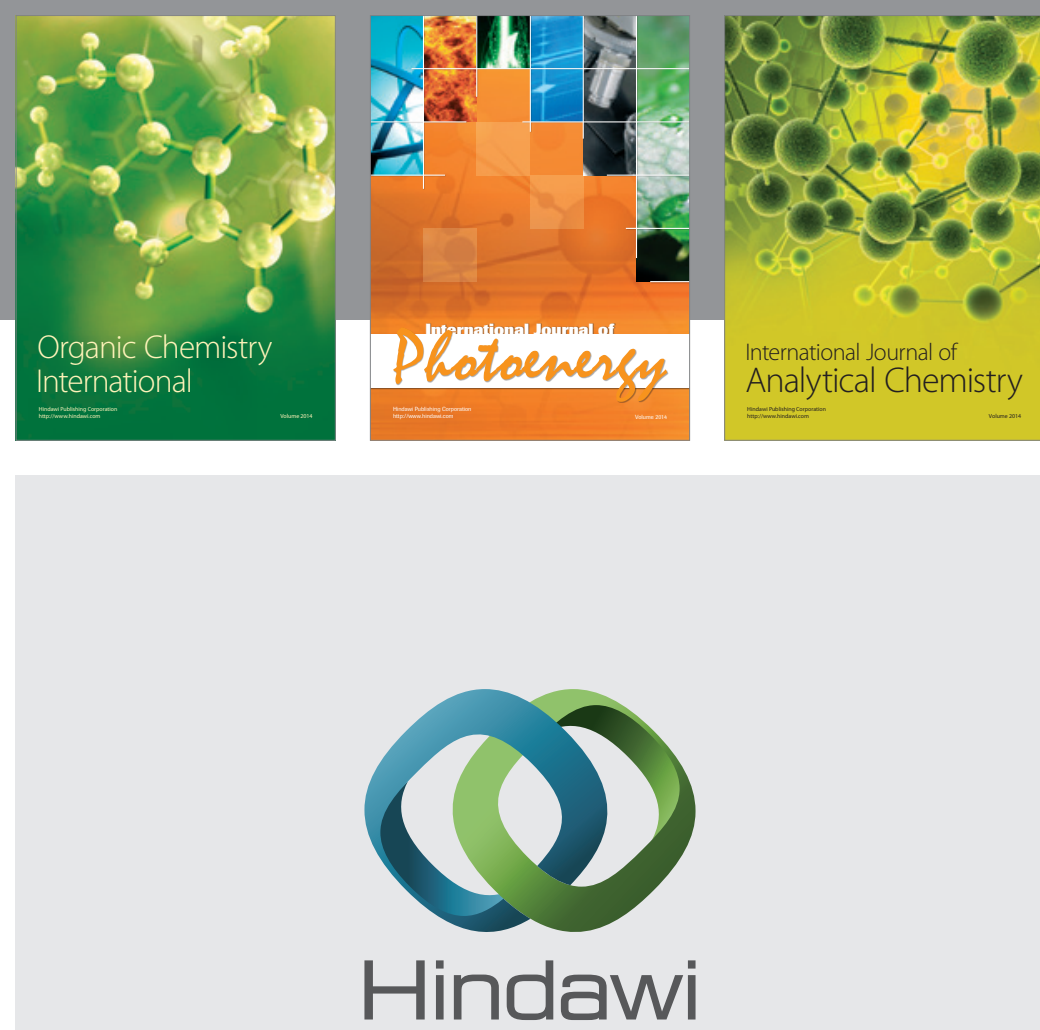

Submit your manuscripts at

http://www.hindawi.com
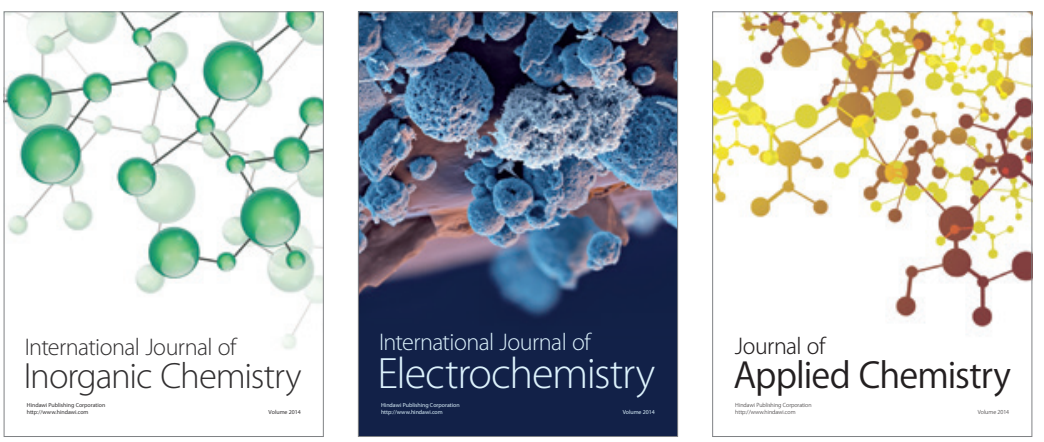

Journal of

Applied Chemistry
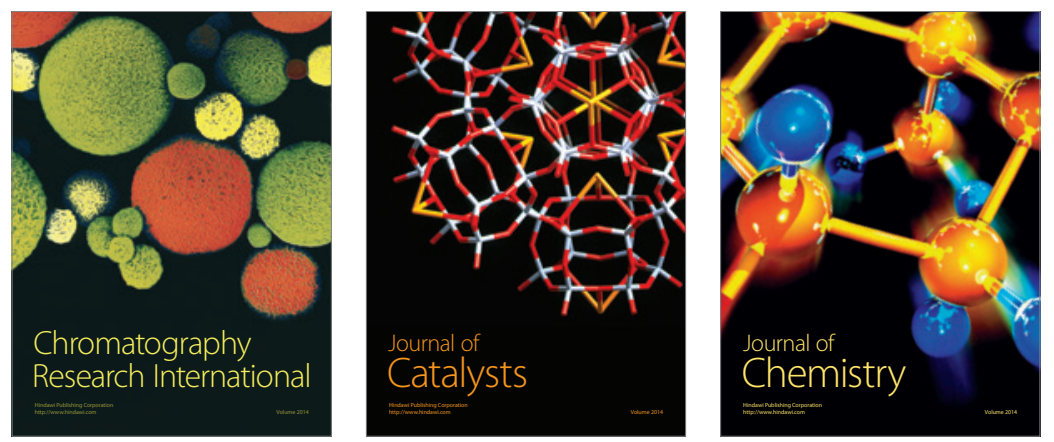
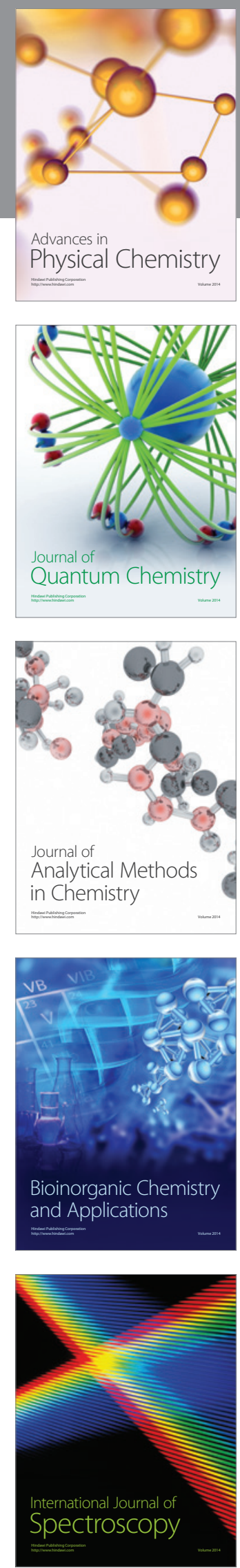